\title{
Molecule Activity Analysis in Ground State of para-Halogenated Diphenyl Ethers Based on DFT Calculation
}

\author{
Long Jiang a, Yue Guo ${ }^{b}$, Qing $\mathrm{Li}^{\mathrm{c}}$ \\ North China Electric Power Research Institute Co Ltd. Beijing 100045, China. \\ akaveykikiy@163.com, bhosa@163.com, c13910680082@139.com
}

\begin{abstract}
Taking diphenyl ether (DE) as the reference, this paper has calculated the ground state orbital energy of DE and other 9 para-halogenated diphenyl ethers via the Gaussian 09 software to discuss the molecule activity on the ground state from views of the gain/lost electron abilities and transition. Then, the time-dependent density functional theory (TD-DFT) at the same level was used to calculate the ultraviolet spectra of them. From obtained conclusions above, the effects on the UV spectrum (ground-excited state electron transition) and the ground state abilities of the introduction of different halogen substituents for para-halogenated diphenyl ethers were explored. Results show that: $\mathrm{DE}$ is most vulnerable to electrophilic substances in the ground state, meanwhile, CDE-15 and BDE-15 are vulnerable to nucleophilic substances to lose electrons; the introduced para-halogen substituent can reduce the energy gap of $\mathrm{DE}$, the increasing atomic volume of substituents always increases the maximum absorption wavelength and absorption intensity of DE.
\end{abstract}

Keywords: Time-dependent density functional method; para-halogenated diphenyl ethers; ground state molecule activity; UV spectrum; electron transition.

\section{Introduction}

As typical halogenated phenyl ether, polybrominated diphenyl ether (PBDEs) and polychlorinated diphenyl ether (PCDEs) were widely used as flame retardant, lubricant and other commercial conducts, causing great harm to the development, genetic system, endocrine system of human beings and organism [1-2]; polyfluorined diphenyl ether (PFDEs) were mainly used in fluorine pesticide preparation, while presents smaller environmental risk compared with PBDEs and PCDEs. Among them, para-halogenated diphenyl ethers are a type of halogenated phenyl ether because of their stock and harm in the environment [3]. Previous researches about para-halogenated diphenyl ethers only focused on the processes of degradation, transformation, and the effect of different halogen substituents on the structure and vibration spectra, but lacked of the deeper analysis of molecular activity and transition mechanism [4-5]. In this paper, we select ten para-halogenated diphenyl ethers as target compounds: FDE-15, BDE-15, FBDE, CBDE, FCDE, CDE-15, FDE-3, BDE-3, CDE-3, and DE. The aim of this paper is to explore the effects on the UV spectra (ground-excited state electron transition) and the ground state abilities of the introduction of different halogen substituents for para-halogenated diphenyl ethers.

\section{Methods}

Using the density functional theory (DFT) embedded in Gaussian 09, the optimal structures of 9 kinds of para-halogenated diphenyl ether and diphenyl ether (DE) were calculated at the level of B3LYP/6-31G(d). Based on the obtain optimal structures, the frontier molecular orbital energy of each congener was calculated to evaluate the molecule activity of the ground state from views of electronic transition ability and the abilities of gaining and losing electronic. The UV spectra of ten congeners were calculated at the same level via time-dependent density functional theory (TD-DFT) at last [6]. 


\section{Results and Discussion}

\subsection{Ground State Molecule Activity Analysis of para-Halogenated Diphenyl Ether}

The environmental stability of compound is not only determined by its own physical and chemical properties, but also by the inductions of UV irradiation, microbial degradation and other conditions. Ionization energy and electron affinity can reflect the abilities of gaining/losing electron, that is mean the degree of difficulty to react with electrophilic/nucleophilic substance. The ionization energy of compound is relative with the level of occupied molecular orbital: the higher of the highest occupied molecular orbital (HOMO) level, the larger the first ionization energy, the more difficult to lose electrons. Meanwhile, the electron affinity is relative with the level of unoccupied molecular orbital: the lower of the unoccupied orbital (LUMO) level, the smaller the first electron affinity and the easier to get electrons $[7,8]$.

From Table 1 we can see: the HOMO energy of CDE-15 is the lowest and DE is the largest; with the aspect of the Mulliken charge distribution in different atoms, there are the same charge for atoms distributed symmetrically in two benzenes of DE, FDE-15, CDE-15, BDE-15, because of their symmetric molecule structures (only list the atom charges of left benzene ring in Table 1). For CDE-15, the most negative charge was located in $\mathrm{Cl}$ atom, resulting in the smallest electrostatic repulsion to the outer electron of $\mathrm{Cl}$ atom and the largest electrostatic attraction to the electron of $\mathrm{H}$ atom. In general, congeners with more substituents have more difficult to lose electron; the introduction of $\mathrm{Cl}$ atom is propitious to react with nucleophiles for DE; the introduction of any halogen substituent can weaken the reactive activity of DE with electrophilic substances.

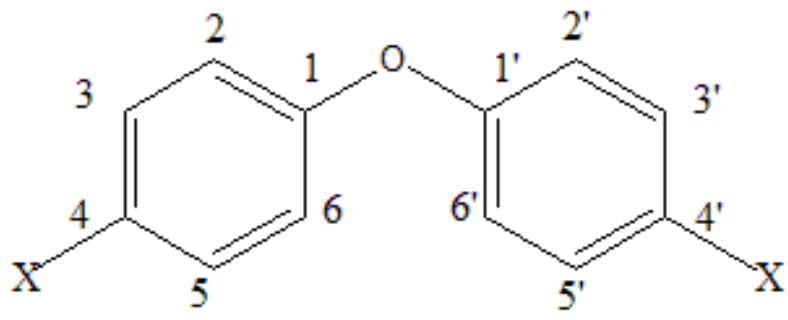

Fig. 1 Molecule geometry and atom mark number of para-halogenated diphenyl ethers

Table 1 Frontier molecular orbital energy level and charge distribution of para-halogenated diphenyl ethers and DE

\begin{tabular}{|c|c|c|c|c|c|c|c|c|c|c|}
\hline Compound & $E_{\text {Номо }}(\mathrm{eV})$ & $E_{L U M O}(\mathrm{eV})$ & $\Delta E(\mathrm{eV})$ & $\mathrm{H} 2 / 2^{\prime}$ & $\mathrm{H} 3 / 3^{\prime}$ & $\mathrm{X} 4 / 4^{\prime}$ & $\mathrm{H} 5 / 5^{\prime}$ & H6/6' & $\mathrm{O}$ & C4/4' \\
\hline $\mathrm{DE}$ & -0.2163 & -0.0083 & 0.2080 & 0.142 & 0.134 & 0.129 & 0.134 & 0.148 & -1.045 & -0.102 \\
\hline \multirow{2}{*}{ FDE-3 } & \multirow{2}{*}{-0.2183} & \multirow{2}{*}{-0.0179} & \multirow{2}{*}{0.2004} & 0.152 & 0.152 & -0.300 & 0.153 & 0.157 & \multirow{2}{*}{-0.577} & 0.381 \\
\hline & & & & 0.142 & 0.134 & 0.130 & 0.135 & 0.147 & & -0.129 \\
\hline \multirow{2}{*}{ CDE-3 } & \multirow{2}{*}{-0.2214} & \multirow{2}{*}{-0.0197} & \multirow{2}{*}{0.2017} & 0.152 & 0.159 & -0.029 & 0.159 & 0.158 & \multirow{2}{*}{-0.574} & -0.066 \\
\hline & & & & 0.144 & 0.137 & 0.132 & 0.138 & 0.149 & & -0.127 \\
\hline \multirow{2}{*}{ BDE-3 } & \multirow{2}{*}{-0.2203} & \multirow{2}{*}{-0.0196} & \multirow{2}{*}{0.2007} & 0.152 & 0.158 & -0.136 & 0.158 & 0.159 & \multirow{2}{*}{-0.574} & 0.083 \\
\hline & & & & 0.144 & 0.137 & 0.133 & 0.137 & 0.149 & & -0.127 \\
\hline FDE-15 & -0.2195 & -0.0234 & 0.1961 & 0.152 & 0.152 & -0.299 & 0.152 & 0.154 & -0.579 & 0.380 \\
\hline CDE-15 & -0.2264 & -0.0283 & 0.1980 & 0.154 & 0.161 & -0.023 & 0.161 & 0.159 & -0.573 & -0.065 \\
\hline BDE-15 & -0.2246 & -0.0282 & 0.1964 & 0.155 & 0.160 & -0.130 & 0.161 & 0.160 & -0.574 & 0.084 \\
\hline \multirow{2}{*}{ FCDE } & \multirow{2}{*}{-0.2238} & \multirow{2}{*}{-0.0262} & \multirow{2}{*}{0.1976} & 0.154 & 0.154 & -0.297 & 0.155 & 0.157 & \multirow{2}{*}{-0.576} & 0.383 \\
\hline & & & & 0.152 & 0.159 & -0.028 & 0.159 & 0.157 & & -0.066 \\
\hline \multirow{2}{*}{ FBDE } & \multirow{2}{*}{-0.2227} & \multirow{2}{*}{-0.0262} & \multirow{2}{*}{0.1965} & 0.154 & 0.155 & -0.297 & 0.155 & 0.157 & \multirow{2}{*}{-0.576} & 0.384 \\
\hline & & & & 0.152 & 0.158 & -0.134 & 0.158 & 0.157 & & 0.083 \\
\hline \multirow{2}{*}{ CBDE } & \multirow{2}{*}{-0.2255} & \multirow{2}{*}{-0.0280} & \multirow{2}{*}{0.1975} & 0.154 & 0.161 & -0.023 & 0.162 & 0.159 & \multirow{2}{*}{-0.573} & -0.065 \\
\hline & & & & 0.155 & 0.160 & -0.130 & 0.161 & 0.159 & & 0.084 \\
\hline
\end{tabular}

In addition, the energy different $(\Delta \mathrm{E})$ between the HOMO energy and LUMO energy always reflects the excitation activity and spectrum absorption of compound [18]. Table 1 has also shown the $\Delta \mathrm{E}$ of ten congeners, and the sequence of $\Delta \mathrm{E}$ for them was: FDE-15 $<\mathrm{BDE}-15=\mathrm{FBDE}<\mathrm{CBDE}<$ FCDE $<$ CDE-15 < FDE-3 < BDE-3 < CDE-3 < DE. Due to the introduction of para-halogen 
substituents, the $\Delta \mathrm{E}$ of $\mathrm{DE}$ becomes narrow, and the ground state is more vulnerable to be motivated into the excited state (the decline of ground stability).

\subsection{UV Spectra Analysis of para-Halogenated Diphenyl Ether}

Fig. 2 shows the calculated UV spectra of nine para-halogenated diphenyl ether and DE. Ten congeners can be divided into three categories: the first category contains BDE-15, CBDE and $\mathrm{CDE}-15$, which all have two substituents $\left(\mathrm{Cl}\right.$ or $\mathrm{Br}$ ) and possess the max $\lambda_{\max }$ and adsorption intensity among ten congeners, concluding that the more of the $\mathrm{Br}$ atoms and the larger of the substituents always increase the $\lambda_{\max }$; the second category contains BDE-3, CDE-3, FBDE, FCDE (ordered by $\lambda_{\max }$ ), the $\lambda_{\max }$ of BD-3 and CDE-3 are larger than FBDE and FCDE manifests that the introduction of $F$ atom is not conducive to the excitation of DE; the third category contains DE, FDE-3 and FDE-15, and the $\lambda_{\max }$ and UV spectra of them are similar. By comparing the UV spectra of ten congeners comprehensively, more and larger para-halogen substituents always promote the electron excitation transition of DE.

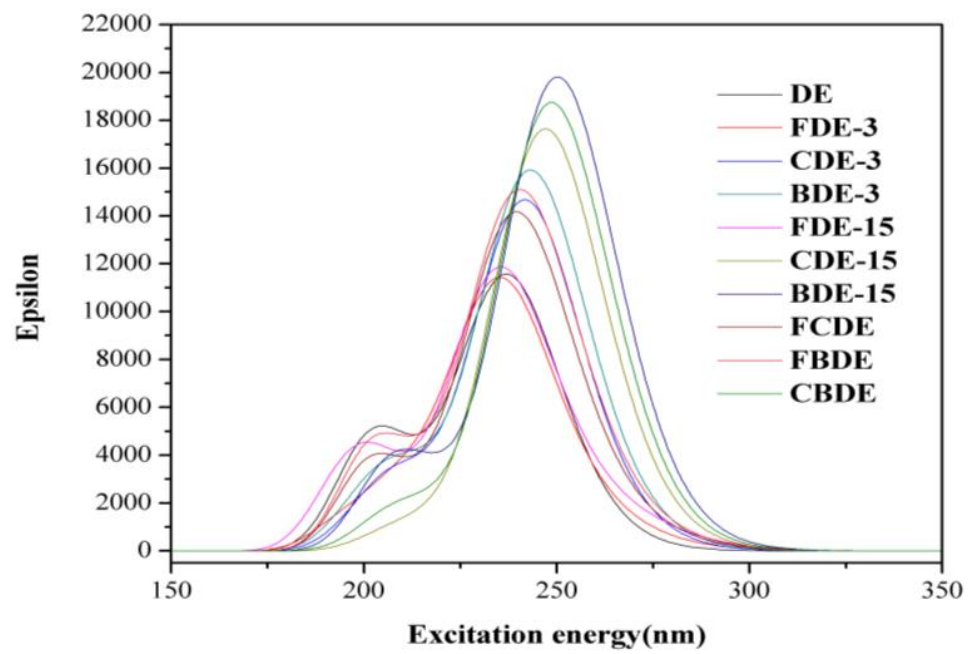

Fig. 2 UV spectra of para-halogenated diphenyl ethers and DE

\section{Summary}

In the ground state, DE can react with electrophilic substances easiest and gain electron, while CDE-15 can react with nucleophilic substances easiest and lose electron, that is because the introduction of para-halogen substituents narrow the energy gap between the HOMO and LUMO of DE, resulting in the excitation transition of DE. Except CDE-15 and DBDE, other congeners have two adsorption peaks in UV spectra, and the $\lambda_{\max }$ increase along with the number and the volume of para-halogen substituents.

\section{References}

[1] P. R. Erickson, M. Grandbois, W.A. Arnold, Photochemical formation of brominated dioxins and other products of concern from hydroxylated polybrominated diphenyl ethers (OH-PBDEs), Environ. Sci. Technol. 46 (2012) 8174-8180.

[2] I. Labunska, S. Harrad, D. Santillo, Domestic duck eggs: an important pathway of human exposure to PBDEs around e-waste and scrap metal processing areas in Eastern China, Environ. Sci.: Processes Impacts. 15 (2013) 503-511.

[3] J. Chevrier, K.G. Harley, A. Bradman, Polybrominated diphenyl ether (PBDE) flame retardants and thyroid hormone during pregnancy, Environ. Health Persp. 118 (2010) 1444-1449.

[4] J. Zhou, J.W. Chen, C.H. Liang, Quantum chemical investigation on the mechanism and kinetics of PBDE photooxidation by 3OH: a case study for BDE-15, Environ. Sci. Technol. 45 (2011) 4839-4845. 
[5] S. S. Qiu, X.H. Tan, K. Wu, Experimental and theoretical study on molecular structure and FT-IR, Raman, NMR spectra of 4, 4'-dibromodiphenyl ether, Spectrochim. Acta A. 76 (2010) 429-434.

[6] D. Guillaumont, S. Nakamura, Calculation of the absorption wavelength of dyes using time-dependent density-functional theory (TD-DFT), Dyes Pigments. 46 (2000) 85-92.

[7] Q. Zou, L. Jiang, X. Y. Du, Optimization of phenanthrene structure and vibrational spectra studies based on density functional theory, Chin. J. Lumin., 33 (2013) 3151-3156.

[8] L. Fang, J. Huang, G. Yu, Photochemical degradation of six polybrominated diphenyl ether congeners under ultraviolet irradiation in hexane, Chemosphere. 71 (2008) 258-267. 